\title{
Exploring Human Mental Models of Robots through Explicitation Interviews
}

\author{
Dag Sverre Syrdal, Kerstin Dautenhahn, Kheng Lee Koay Michael L. Walters, Kerstin Dautenhahn, \\ and Nuno R. Otero
}

\begin{abstract}
This paper presents the findings of a qualitative study exploring how mental models of a mechanoid robot using dog-inspired affective cues behaviour emerges and impacts the evaluation of the robot after the viewing of a video of an assistive robotics scenario interaction with the robot. It discusses this using contrasting case studies based on the analysis of explicitation interviews with three participants. The analysis suggests that while for some users zoomorphic cues may aid in initial interactions, they need to be framed in an authentic interaction, highlighting the actual capabilities of the robot as a technological artifact, and how these impact the everyday life and interests of the potential user.
\end{abstract}

\section{INTRODUCTION}

$\mathrm{T}$ his paper explores qualitatively the specifics of how a mental model of a robot is formed.

The term mental model is here derived from [1] and understood to be a process, wherein a mental model is constructed from mental representations of objects and process in the external world. This can then be used to predict outcomes of events and behaviour in the physical world. While the relationship between the representations contained in the model and their external world counterparts need not be one of direct correspondence (i.e. they may not accurately reflect reality), and the processes may not be consciously apparent to the individual at all given times, the contents of the model should be expressible through verbal and 'folk scientific' statements by the holder of the model. Mental models are important to human-robot interaction (HRI) since they act as a reasonable predictor of interactions with, and evaluations of, a given system. In order to investigate these issues an exploratory study into mental models in HRI has been performed.

Approaches in the literature to the study of human mental model of robots in HRI can be grouped in two rough categories:

The first is the study of 'low-level' mental models for human beliefs regarding very specific categories. For

Manuscript received March 1, 2010. This research was conducted as part of the EU Integrated Project LIREC (LIving with Robots and intEractive Companions) and funded by European Commision FP7-ICT under contract FP7-215554.

D.S. Syrdal, K. Dautenhahn, K.L.Koay, M.L.Walters are with the Adaptive Systems Research Group, School of Computer Science, University of Hertfordshire, Hatfield, Herts, UK; \{d.s.syrdal: k.dautenhahn; k.1.koay; m.l.walters\}@herts.ac.uk

N.R. Otero is with the Department of Information Systems, University of Minho, Guimaraes, Portugal; nuno.otero@dsi.uminho.pt instance, Fischer and Lohse [2] describe an investigation into human mental models of a robot's situation awareness and suggest means of modifying such models to ones that are more in line with the robot's actual capabilities.

The other approach is looking at 'higher-level' mental models drawing on metaphor for their conception. In particular, several authors [3-5] have examined the role of anthropomorphic mental models where robots have been rated according to personality traits appropriate for humans as well as traits that are more appropriate for mental models that would see the robot as purely machine-like. Kiesler \& Goetz [4] found a link between aspects of a participant's anthropomorphic mental model and their ability to cooperate with a robot. Syrdal et al. [6] found an effect in which attributions associated with a more anthropomorphic mental model of a robot had an impact on how participants responded to and evaluated the proxemic (social distances towards a human) behaviour of a robot. Furthermore, Andonova [7] examined how a high-level mental model of a wheelchair robot could be influenced and changed.

High-level mental models of robots, which incorporate anthropomorphic dimensions of interaction with robots, have been utilised in the design of robots (for instance,

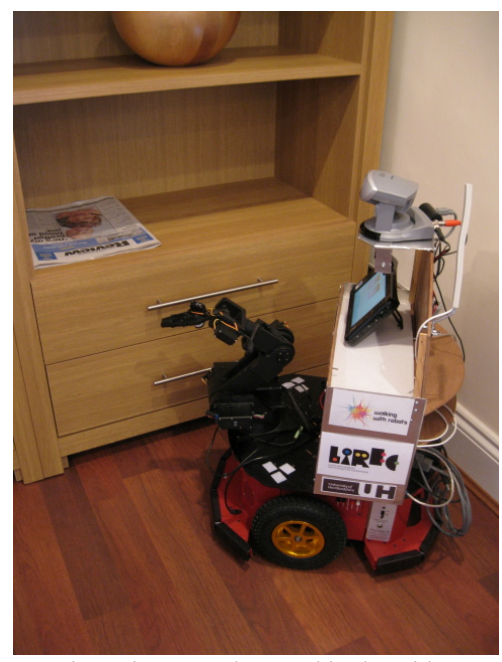

Fig 1 Pioneer Robot used in the Video

Walters et al. [8] and Breazeal [9]). This use of anthropomorphic cues and interaction modes have been implemented for two main reasons: The first is to facilitate interactions that seem natural to the human interactants by drawing upon existing mental models of expected 
interaction, thus reducing the effort needed by the human interactant. Also, the ability to draw upon an existing body of knowledge is important, both in terms of "commonsense" as well as findings from the social sciences regarding mental models of expected behaviour in given interactions. In this sense, the use of anthropomorphic cues is no different from the practice in HCI (human-computer interaction) of incorporating features of popular existing software packages in new interfaces or conceptualizing interactions through the use of metaphors (the common example is the desktop metaphor [10]). However, pitfalls of the use of anthropomorphic cues have been highlighted. For example, if the appearance and cues of the robot seem to fit too well within a mental model in which the robot is understood as human-like, and if human behaviour within an HRI scenario based on such a model does not elicit the appropriate response from the robot, this may lead to a feeling of disillusionment and rejection of the robot for the user [11]. A reasonable conclusion here is that the appearance and cues displayed by the robot should be familiar enough to the user that the user is capable of discerning the intentions of the robot, while not engendering unrealistic assumptions of the anthropomorphic nature of the robot.

One method which has been applied is that of attempting to substitute anthropomorphic mental models with ones that are zoomorphic in origin. This has been the case for the entertainment robots such as Sony's AIBO and Ugobe's Pleo, as well as the eldercare robot PARO [12]. Nicolescu and Mataric' work with using dog-like cues to direct attention with a Pioneer robot is also an excellent example of this.[13] The increasingly widespread use of robots using zoomorphic appearance and cues warrants a reexamination of the higher-level mental models that interactants may have of robots. While there is evidence that personality attributions mainly associated with anthropomorphic mental models may be suitable for nonhuman animals $[14,15]$, and there are standardized ways of measuring such attributions for specific species [16], evidence suggests that human mental models of animalinspired robots differ from those of the animal that the robot is based on [17].

\section{METHODOLOGY}

\section{A. Aims}

The above suggests that an in-depth investigation into how mental models are formed, shaped and then influence how participants evaluate a robot is a useful avenue of study which uses qualitative methods. This approach allows for an understanding of mental models that is data-driven, and could allow us to examine the participants' models on their own terms rather than the more narrow lenses that quantitative methods necessitate (for example the predefined semantic scales based on human personality traits in [3-6]). The study presented here aimed to examine and explore these issues in contrasting case studies, using interview transcripts from three participants. The case study approach has previously been used in HRI studies which have aimed for in-depth exploration of human perceptions of robots [18]. This methodology is not intended to replace quantitative methods, but rather aims to complement such methods, by allowing the researchers to get an in-depth understanding of the reasoning that leads to particular quantitative results as well as open up new avenues of investigations by raising new possible research questions.

\section{B. The Transcripts and Video}

The interview transcripts were obtained from a study performed for the purpose of evaluating the usefulness of affective cues inspired by dog-behaviour for wider use within the European LIREC project [19]. One of the purposes of these interviews was to pilot the display of the cues as well as to elicit responses that would allow the researchers to create a quantitative questionnaire based on the description of the participants. These results, along with the results from a quantitative pilot study are currently being written up for publication.

The video used in this study showed a user and a guest (named Anne and Mark) interacting with a robot that used affective non-verbal cues. The behavioural cues created to be exhibited by the robot were not identical to, but were inspired by, cues used by dogs interacting with humans in the same situations. The video was created at the University of Hertfordshire Robot House, with input from a group of ethologists from the Ethology Department at Eötvös Loránd University (Budapest). The motivation for the study was that if these cues were effective, they would elicit mental models of the robot and its behaviour that would draw upon existing mental models of dogs and dogbehaviour. As such, its use would allow us to investigate zoomorphic mental models in detail. An overview of the video follows in table 1.

\begin{tabular}{|c|c|}
\hline Scene No. & Brief Description \\
\hline 1 & $\begin{array}{l}\text { Robot/Dog is in dining room, Owner enters from } \\
\text { outside, robot greets owner. }\end{array}$ \\
\hline 2 & $\begin{array}{l}\text { Robot/Dog is in dining room, Guest enters from } \\
\text { outside, robot/dog greets guest and uses social } \\
\text { referencing to interact with owner. }\end{array}$ \\
\hline 3 & $\begin{array}{l}\text { Robot/Dog follows owner to the kitchen and is } \\
\text { loaded with items for tea and biscuits. }\end{array}$ \\
\hline 4 & $\begin{array}{l}\text { Robot/Dog attempts to gain guests attention for } \\
\text { help in unloading. }\end{array}$ \\
\hline
\end{tabular}


Owner and Guest have tea and converse with robot /dog watching.

Guest leaves, robot/dog engages in "farewell" behaviour with guest.

The robot used in the video was a Pioneer $^{1}$ (see fig 1) which is mechanical-like in appearance, approximately the same size as a medium size dog, but in other respects was not particularly dog-like.

\section{The Behaviours Used}

The behaviours across this video were intended to be analogous of that of dogs, while taking into account the different modalities for sensing and communication. For instance, if a dog in a given situation would use its sense of smell to examine something, the robot would instead appear to be examining something by moving its camera.

The greeting behaviour for the owner consisted of the robot moving towards the owner as she entered, orienting its camera briefly towards the face of the owner and then moving away in the direction the owner would later move towards. This behaviour was intended to communicate enthusiasm both in terms of greeting the owner and aiding in the tasks the owner was later to perform.

The greeting behaviour for the guest was similar, however, here the robot spent more time on examining the guest, and immediately turned to the owner for the purposes of social referencing [20] by orienting its camera to the owner when the owner appeared..

The "farewell" behaviour towards the guest consisted of the robot orienting its body and camera towards the guest, observing him as he left the room. The "farewell" behaviour towards the owner consisted of the robot orienting its body towards the owner and then moving towards the owner as she walked to the door, following the owner to the door, only stopping as it reached the door.

The underlying "story arc" of the video was that of a friend visiting the owner of a robot who primarily used it as a moving platform for transporting objects from place to place. This task, like the behaviours, were inspired by actual tasks performed by helper-dogs for the disabled.

\section{Participants}

Three participants were used in this study. The participants were chosen primarily in order to highlight three different approaches to the robot's behaviour in the video.

Two of the participants used in this study were both male, in their mid-twenties and post-graduate education.

Participant BH1 comes from a science background working towards as $\mathrm{PhD}$ in one of the physical sciences

1 Commercially available robot platform from MobileRobots MobileRobots ( http://www.activrobots.com/ ) and is highly proficient with computers, capable of coding programs for data collection and analysis within his field. His family has owned several dogs.

Participant TE1 comes from a computer science background, and is currently working towards a $\mathrm{PhD}$ in the subject and has experience with robotics. He does not own a dog, nor does his immediate family.

Participant NB1 is a female in her mid-forties. She is a stay-at-home mothers. And has suffered from debilitating arthritis since an early age While there may be a temptation to refer to her as technically naïve due to her lack of formal training in the use of computing equipment, she uses computers extensively in her day to day life, and before the interview made references to her experience of voice recognition software that she attempted to use as a substitute for typing, which can be painful due to the arthritis. She has previously interacted with a robot in a proxemics study similar to that reported in [21] She does not own a dog.

The interviews from these three participants were chosen from a larger pool of interviews which had been conducted to create a quantitative questionnaire for further use of the video. The two male participants were chosen due to their clear membership in the "early adopter" demographic for consumer electronics, like personal robots. This along with their different backgrounds in terms of technical experience of robots as well as differences in exposure to dogs, was hoped to illustrate different aspects of how mental models would form.

The third participant was chosen as a contrast to the previous two. She does not have their extensive experience of using computers, but have had experience in using particular technical aids for overcoming problems arising from her arthritis.

It was hoped that this combination of backgrounds would elicit and highlight salient issues in this investigation.

\section{E. Method}

The participants viewed the video and were then asked to participate in an explicitation interview exploring their experience while watching the video. This interview was unstructured, the dialogue mainly focused on a chronological account of the videos as well as requests from the interviewer for elaboration on statements from the participants attempting to draw out as much information regarding the issues raised by the participants, and care was taken not to mention the dog-inspired origin of the behaviour in order to assess the legibility of the cues. Also, while participants were prompted, towards the end of the interview, to compare the robot to something else, this was not done until the end of the interview, and responses to this prompting was recorded and reported as such. We previously used this interview technique in examining 
responses to HRI videos [22]. Explicitation interviews aim to evoke a revivification of the perceptual experience and one of the benefits of this is that it allows the construction of a narrative to be recorded rather than just the endproduct narrative itself [23]. In this way, the technique allows us to examine how the participants describe their experiences and how these descriptions become the building blocks of a narrative in which the mental model of the robot emerges The transcripts were analysed in detail using the Grounded Theory approach in interacting with the data [24]. This approach was chosen as its open-ended, data-driven nature was deemed suitable for the exploratory nature of this investigation.

The initial open coding focused on identifying and coding themes relating to how the participants described the behaviour of the robot and the robot itself. Early on in this process, the salient themes became those relating to attribution of agency, emotive descriptions, referencing of personal experience, descriptions of robot behaviour, and the use of metaphor in describing the robot. This was followed by axial coding, in which the initial themes, and their relationships with each other, were examined across the transcripts of the participants.

\section{RESULTS:}

\section{A. The main dimensions}

Organising these themes into dimensions yielded two primary dimensions in which the two transcripts differed; that of describing the robot using the dog metaphor and understanding the robot as a malleable, customizable, technological artefact. The way that these two dimensions interacted to form the particulars of the two participants' mental models of the robot and subsequent evaluation of the robot, became the focus of the analysis.

\section{B. Describing the robot-Dog metaphor and the robot's mechanical nature:}

BH1, when describing the robot's behaviour clearly identifies its behaviour as doglike and frames this behaviour directly within his own personal experience of dogs:

BH1: It acts a bit like a dog, actually, and goes up to investigate who comes through the door.

E: You say it acts a bit like a dog, can you elaborate?

BH1: I got a couple of dogs at home, and as soon as I come through the door or as anybody comes through the door they get up and investigate who comes into the door by walking up to the person and have a look.

This is quite important for the development of the rest of the interview, as this participant continues to frame the behaviour of the robot within interactions that have taken place within his own everyday experience. Of particular interest is the following discussion of the robot's attention seeking behaviour:

BH1: I suppose because it needs to grab his attention and to assess how it should grab his attention, I suppose if it moved fast it would be very useful to grab his attention, although it would hurt him. And as it does not seem to be able to talk or make any sounds at all, it has to sort of assess that it is gonna have to collide with him to grab his attention...

E: ... What do you think of the way the robot tried to grab his attention?

BH1: Well, obviously it assessed that the guest was paying no attention to the robot and then decided that the best course of action would be to sort of gently grab his attention by driving into his foot rather slowly. I would say that was quite acceptable.

This exchange seems to suggest that $\mathrm{BH} 1$ sees the robot as having an agency defined by its task, and the ability to use the modalities provided by its form to compensate for its lack of verbal ability.

NB1, on the other hand, describes the robot in quite mechanical terms. She focuses primarily on low-level descriptions of the behaviours as well as the practical issues for the user:

NB1:It is quite small, whiteish coloured, and it had a small camera, which could move up and down, but it [the robot] was quite short. It followed her to the kitchen, wheeled at a steady pace keeping up with her. Then it stopped. It must have decided that it knew where she was going, and so stopped as it knew where Anne was if she needed it.

NB1: When Mark came in, it moved its camera up and down slowly as if measuring him, maybe, I think, to get a picture of him so that he could be recognized more quickly the next time he was visiting.

These statements indicate a mental model of the robot as a purely mechanical entity, whose function defines its behaviour and motivation. The behaviours are descriped in low-level terms and explained in terms of their utility to perform subsequent tasks. Moving on from this, she highlights the actual task the robot was used for.

NB1: It [the robot] follows her to the kitchen and she loads it with the plates and cutlery and things. I think it's ok, but for that kitchen I think it is a bit too close, She needs to bend over too much when loading it at that angle, I think I would want it a bit further away, if I was to use it.

The robot's behaviour here is critiqued purely in terms of how they meet the users needs in terms of the practical performance of the task. 
This is continued in her description of the attention seeking sequence:

NB1:I am not sure if I liked the way that it acted when Mark was reading his paper and not noticing the owner. I think maybe it shouldn't have bumped into him, maybe used a beep or something to alert him... On the other hand, Mark didn't seem to mind, and since he was allright with it, maybe it doesn't need to be changed.

NB1 here highlights an episode where the robot is acting in a manner that she thinks may be problematic. What is interesting here is that after drawing up possible solutions, using sound based communication, she concludes that the behaviour of the robot was appropriate due to the acceptance of it by the user, another example of her focus on the practical aspect of adopting such a technology.

TE1 in contrast, seems to incorporate aspects of both the above participants, models of the previous participants. When asked to describe the behaviour of the robot, the following exchange occurs:

TE1: Ok, sure, I believe she comes into the room, the robot sees her, and she says something like "Hello Robot", and the robot seems to respond by looking at her but not really responding in ... in any other way.

E: OK, could you think back to the way Anne and the robot move in that particular sequence. Could you describe the way the robot moves?

TE1: Ehm...the robot seems to direct himself towards her, going to towards her and seems to focus his camera on her.

Participant TE1 here draws on his particular technical background to deconstruct the overall behaviour of the robot into a set of sub-behaviours, which are determined by how it uses its camera and movement. However, TE1 still references more high level communicative functions by noting their absence e.g. "...not really responding”.

This reference to the absence of behaviour is repeated in his description of the robot meeting the guest, the robot is described as constrained by its abilities.

TE1: It seems like he does greet him a little bit, but he doesn't have the power to actually go and do some interaction with Mark

When describing the attention seeking behaviour, TE1, like BH1 and NB1 highlights the lack of verbal/auditory communication modalities for the robot.

TE1: Given how its capabilities were that he couldn't make a noise or something, I would say that is the only way he could get his attention.

The responses of the three participants indicate an underlying difference in how the robot is viewed. BH1 has framed the robot and its behaviour within that of his own experience through the similarities with dog-behaviour. Thus, he seems to regard the behaviour of the robot in the attention seeking sequence as an active adaptation on the basis of actively pursuing a task. NB1 proceeds to frame this behaviour within the interactions with and reactions from the users. Interestingly, the more technically minded
TE1, however, sees the situation more clearly from the robot's perspective with the constraints as limiting and the robot as being forced into a set of actions.

\section{The possibility of 'bettering' the robot.}

These limitations are a continuing theme in TE1's considerations of the robot behaviours:

TE1: It seems like acting a little bit socially, but not too much. Most of the time it acted just like a tool for the owner. And...sometimes it did seem try to kind of find a connection with the people, but I don't think...it didn't seem like it succeeded in that.

Here, the robot is described as trying to find a connection to people as it did in the initial discussion of the greeting sequence, but is unable to transcend the constraints of its hardware, by means of particular sounds or verbal utterances, to make this connection. While the affective dimension of TE1's description of the robot is not explicit in the above statement, this dimension is made so along with an acknowledgement of the utility of such a n affective connection, despite its lack of authenticity later on:

TE1: I think so, yeah...if somehow he would seem a little bit attached to me. He would have a reason to help, it would be nice, he would be more than a tool. Then sometimes he is maybe in the way, if you have the feeling that he is doing that because of attachment you would be more lenient with that.

TE1: Yes, I am fully aware that it is really easy to project emotion or something on something that doesn't have it. But still in daily life you don't consider it. With pets for instance you easily project intelligence or emotions on them. It just happens even though you know when you are talking to it, it doesn't understand it. But it is still comforting to project it on it.

Here, the participant references anthropomorphic aspects of interactions with other non-human entities, and while acknowledging that these aspects are one-sided, still confesses to being not only susceptible to them, but also the possibility of drawing emotional support from them. As such, there is a similarity with one of the case studies reported in human-AIBO interactions by Turkle [18], where the human interactant saw the robot not only as a creature with the possibility to have emotions, but also representing an avenue through which she could fulfill her need to nurture another being. Similarly, TE1 sees the possibility of the robot being able to act emotionally, but sees this possibility undermined by the constraints of its current capabilities. Interestingly, TE1 also seems to argue that the robot as well is struggling against these constraints. TE1's reasoning implies that it is possible to remove these constraints, and this removal is mutually beneficial both for the robot (which succeeds in its attempts to '...find $a$ connection.', and '..become more than a tool.'), and the 
user which will be comforted by the robot. In this sense, TE1's sentiments echoes Turkle's Melanie in the need to nurture the robot, albeit through different modalities and a more reasoned approach, not necessarily based on emotional needs, but more likely based on a greater sense of the efficacy of creating a technical solution to aid the robot. It is also interesting to note that when pressed to compare the robot's behaviour to a non-robot entity, TE1 refers to children as well as dogs in his description:

TE1: It reminds me a bit like children. If you don't give them attention, they get busy and run around until you look at them and give them attention.

TE1: ... like children if the mother leaves there is more attachment. The same with dogs if the owner leaves there is more display of this behaviour than with random people.

In contrast, $\mathrm{BH} 1$ focuses less on the robot itself, but rather on its role in the interactions when considering its behaviour:

BH1: It seemed very socially capable really. It reminded me of an animal, particularly a dog in that it wanted to be near its master. Follows its master around the house, it obeyed every command, seemed to understand every command, particularly when Mark walked in and Anne introduced Mark to the robot and the robot to Mark it seemed to acknowledge him. And then just acted normally.

Again, there is a grounding in the participant's experience with dogs to understand the robot as dog-like, which here leads to a favourable assessment of the robot's behaviour. By emulating a dog, the robot allows itself to be slotted into the participant's expectations of possible interactions within domestic settings, and so the robot is considered much more socially capable as it conforms to these pre-existing expectations. As such, the need for nurturing through increased modalities is not present for BH1.

When considering the social aspect of the interaction. NB1 draws from her own experience with assistive technologies:

NB1: It seemed to understand what they were saying to it really easily. I remember trying to use voicerecognition software a couple of years ago, so I wouldn't have to type so much, you know, because of my arthritis, but I don't think it worked that well. I remember thinking it was easier just to type a bit more slowly.

This cements the notion that for NB1 the robot's potential is primarily as a tool to use in particular situations that she may find difficult.

\section{Evaluating the Robot's role in the Situation}

However, when assessing the usefulness of the robot within the scenario presented there is another difference between the three participants:
TE1: I would think so, yeah. It definitely could help carrying stuff like that. It shows enough intelligence to follow people and bring stuff, and I would say that is useful yeah.

NB1: Yes, I could see a use for it. I remember when I broke my leg and had problems walking. I couldn't make myself a cup of tea, and had to wait for my daughter or husband to come home to help me with that. It makes you feel a bit helpless you know. Maybe we could get these on the $N H S^{2}$ ?

BH1: Not particularly [useful], she loaded it with a couple of things, and brought the rest in herself and it seemed like a pointless exercise. She could have done it all herself...I don't think you could have a relationship with it [the robot] the way you could with a dog, cause a dog has a personality, while for a robot, the personality is just a couple of subroutines...

This divergence of opinion can most easily be understood through the perceptions that the participants have displayed throughout the earlier parts of the interview. TE1, having described the robot as constantly being 'frustrated' by its constraints in its efforts to connect with the humans in the scenario, here highlights the one function that the robot seems to be able to perform without such 'frustration'. This leads him to consider the robot's ability on the robot's own terms.

NB1 relates the robot to her own needs and sees it as a potentially useful aid in her everyday life, highlighting an episode where it could have been of use. This is consistent with her situating the robot within the tasks and interactions that previously emerged in the interview.

BH1's comments on the other hand, reflects his ability to insert the robot into what he considers a plausible social scenario. For him, the robot seems capable of functioning in existing interactions, which leads him to focus on how the robot can bring added value to these interactions, a function that the robot fails to perform.

\section{E. Summary}

The analysis of the interview transcripts suggests that $\mathrm{BH} 1$ and TE1 took an interest in and interpreted the zoomorphic cues as communicating emotive information and both referred to dog behaviour when attempting to describe them. The participants differed, however, in how dog-like they saw them. Participant $\mathrm{BH} 1$ repeatedly referenced his own rich experience of dog-behaviour when describing and reasoning about the cues and their purpose, while participant TE1, on the other hand, while referencing dogs, also referenced children as well as attempting to reconcile a more technical deconstruction of the robot's behaviour with the affective dimension of the cues.

\footnotetext{
${ }^{2}$ A reference to the British National Health Service.
} 
This divergence became more apparent as the narrative was constructed within the interview. In the later stages of the interview, the utility of the BH1's dog-based mental model while useful for understanding the robot, also seems to have led him to unfavourable evaluation of the robot and its utility especially when this rich mental model of "the robot as a dog" led to a direct comparison of the robot with a dog.

TE1, on the other hand, while interpreted the robot's behaviour successfully using a mental model still containing dog metaphors, incorporated other aspects into this model, which allowed him to look for means for the robot to overcome its lack of sophistication.

$\mathrm{NB} 1$, in contrast to both the other participants, did not consider the affective communication aspect of the interaction in her descriptions, choosing instead to focus on the task related aspects of the video, and when considering interactions, focused on ease of use as well as acceptability. She also referenced her own experiences with assistive technologies as well as specific instances where the robot as being portrayed in the video would be of use.

\section{CONCLUSIONS}

The responses from these participants highlight important issues in dealing with the robot portrayed in the scenario:

One the one hand, there is the approach exemplified by $\mathrm{BH} 1$. In this approach the behaviour of the robot is not only taken as offered by the scenario, but is easily incorporated into this participant's own experiences and expectations. The robot is understood more in terms of its role as a "dog", or even possibly as a dog-substitute. While initially this approach seems to make the participant more appreciative and accepting of the robot, the placement of the robot in this role, also puts demands on the robot, both socially and emotionally, as well as in terms of usefulness. In this case, the robot fails to live up to some of these expectations.

NB1's mental model of the robot, is not so much considering the robot as a separate entity, but rather focuses on how the presence of the robot impacts her mental models on how tasks are performed. The robot is represented mainly as the sum of its functions. The affective communication aspect of the interaction was completely overshadowed by the use-possiblities that this participant envisaged.

TE1, on the other hand exemplifies an approach in which the robot is considered to occupy a paradoxical position. On one hand, it is seen as a tool, and this approach explicitly acknowledges its 'mechanical', non-human nature. However, it is also imbued with what could be described agency, a purpose to transcend the constraints of its current form. This approach opens up the possibility of a more active user, who sees the robot more of a hobby, a project in which there is a reciprocal relationship between the robot who is given further modalities in which it can communicate and connect with its user, and the user who will enjoy and be comforted by the robot's increasing ability to interact.

The contrast between NB1 and TE1 provide an interesting illustration of the two demographics highlighted in Sung et al [25] where many users who don't fit into the early adopter stereotype acquire consumer robots to perform particular tasks, while some users purchase consumer robots in order to improve and customise them. The perspectives of these users allow for an understanding of how views on the robot in itself as well as their impact on their environment are formed.

While it is certainly easy to argue that it may be difficult to make strong claims about the generalisability of the participants' reasoning to a wider group of users, it is important to note that what this method allows for, however, is to present an in-depth account of how these participants reasoning, attitudes and feelings towards the robot emerge. As such, it provides insights that irrespective of participant numbers can be used in the design of robot technologies, For instance results such as these are very valuable for developing "user personas" in an Interaction Design approach like the one described in [26]. They also raise issues that may be studied further, both qualitatively and quantitatively, for instance, studies such as [27] rely on a highly detailed and sophisticated user profile, which in turn opens the door for customization and personalization of artifacts.

In terms of lessons for further research on the use of zoomorphic and anthropomorphic cues to regulate interactions and emergent relationships, these interviews highlight salient differences in how the nature of companionship with a robot is interpreted and understood by different people and how these interpretations arise and develop. Implications of this work include:

Firstly, anthropomorphic and mechanical reasoning regarding the robot are not necessarily antagonistic to each other, and as the appearance of the robot in this study was not doglike at all, does not necessarily rely on appearance.. This is exemplified by TE1's reasoning regarding the robot incorporates both a sophisticated interpretation of the robot's behaviour which explicitly and knowingly utilises his own anthropomorphic biases while still retaining a core understanding of the robot as a machine. This is reminiscent of the phenomena of Joint Pretense discussed by Clark [4] in regards to interactions with virtual partners, or the Performed Beliefs reported in Pleo blogs by Jacobsson [28]. These conflicting notions of the robot's nature are complementary, and it is telling that in TE1's responses, the anthropomorphic aspects of his reasoning about the robot are most apparent when he considers its 
technical limitations as a robot.

Secondly, it is important to consider the highly important role of personal experience when reasoning about the robot. Dog ownership seems to form the core around which BH1's perception of the robot is constructed, while the experience of being disabled allows NB1 to situate the robot very clearly in her everyday life. The Computer Science background of TE1 seems to create a focus on the specific technical problems that the robot has in performing the social aspects of its tasks. This suggests that while zoomorphic cues may aid in initial interactions for some users, they need to be framed in an authentic interaction, highlighting the actual capabilities of the robot, and how the individual user may relate to and utilise it within their own everyday experience.

\section{V.ACKNOWLEDGEMENTS}

The authors would like to thank the Ethology Department at Eötvös Loránd University, in particular Adam Miklosi, Marta Gacsi Enikö Kubinyi and Gabriella Lakatos for their aid in creating the videos used for this study. We would also like to thank the participants who volunteered to take part.

\section{REFERENCES}

[1] P. N. Johnson-Laird, "Mental Models, Deductive Reasoning, and the Brain," in The Cognitive Neurosciences, M. S. Gazzaniga, Ed. Cambridge, MA: MIT Press, 1994.

[2] K. Fischer and M. Lohse, "Shaping Naive Users' Models of Robots' Situation Awareness," IEEE International Symposium on Robot and Human Interactive Communication (Ro-man 2007), Jeju Island, Korea, pp. 534-539, 2007.

[3] S. R. Fussell, S. B. Kiesler, L. D. Setlock, and V. Y. H. 145-152, "How people anthropomorphize robots.," The Third ACM/IEEE HumanRobot Interaction Conference (HRI08), 12-15 March 2008, Amsterdam, the Netherlands, pp. 145-152, 2008.

[4] S. Kiesler and J. Goetz, "Mental Models and Cooperation with Robotic Assistants," Proceedings of the ACM CHI'02 Conference on Human Factors in Computing Systems, pp. 576-577., 2002.

[5] S. Lee and S. Kiesler, "Human mental models of humanoid robots," In Proceedings of the 2005 international conference on robotics and automation (ICRA 05) (pp. 2767-2772), Barcelona, Spain, 2005.

[6] D. S. Syrdal, K. Dautenhahn, M. L. Walters, and K. L. Koay, "Sharing Spaces with Robots in a Home Scenario - Anthropomorphic Attributions and their Effect on Proxemic Expectations and Evaluations in a Live HRI Trial," Proc. AAAI Fall 2008 Symposium "AI in Eldercare: New Solutions to Old Problems" November 7-9, Washington, DC, USA, 2008.

[7] E. Andonova, "Changing Mental Models of a Wheelchair Robot," Proceedings of the Workshop on How People Talk to Computers, Robots, and other Artificial Communication Partners, Bremen 2006, pp. 131-139, 2006.

[8] M. Walters, D. S. Syrdal, K. Dautenhahn, R. T. Boekhorst, K. L. Koay, and S. Woods, "Avoiding the Uncanny Valley - Robot Appearance, Personality and Consistency of Behavior in an Attention-Seeking Home Scenario for a Robot Companion," Autonomous Robots, vol. 24, pp. 159-178, 2008.

[9] C. L. Breazal, Designing Sociable Robots. Cambridge, Massachusetts: MIT Press, 2002.

[10] J. M. Carroll and J. C. Thomas, "Metaphor and the Cognitive Representation of Computing Systems," IEEE Transactions on Systems, Man, and Cybernetics, vol. 12, pp. 107-116, 1982.
[11] K. Dautenhahn, "Design spaces and niche spaces of believable social robots," Proceedings of the 11th IEEE International Workshop on Robot and Human Interactive Interactive Communication(RO-MAN 2002), Berlin, Germany Sept 25-27, 2002, 2002.

[12] T. Shibata, K. Wada, T. Saito, and K. Tanie, " Human interactive robot for psychological enrichment and therapy.," Proceedings of the AISB '05 Symposium on Robot Companions: Hard Problems and Open Challenges in Robot-Human Interaction Hertfordshire, England, April 12-15, 2005). Hertfordshire, England: University of Hertfordshire, 2005, 98-109., 2005.

[13] N. Nicolescu, and M. J. Mataric, "Linking Perception and Action in a Control Architecture for Human-Robot Domains," Proceedings Thirty-Sixth Hawaii International Conference on System Sciences, HICSS-36, Hawaii, USA, January 6-9, 2003. 2003.

[14] S. D. Gosling and O. P. John, "Personality Dimensions in Nonhuman Animals: A Cross-Species Review," Current Directions in Psychological Science, vol. 8, pp. 69-75(7), 1999.

[15] S. D. Gosling, V. S. Y. Kwan, and O. P. John, "A dog's got personality: A cross-species comparative Approach to personality judgments in dogs and humans.," Journal of Personality \& Social Psychology, vol. 85, pp. 1161-1169., 2003.

[16] E. Kubinyi, B. Turcsan, and Á. Miklósi, "Dog and owner demographic characteristics and dog personality trait associations," Behavioural Processes, vol. 81, pp. 392-401, 2009.

[17] B. Friedman, P. H. Kahn, and J. Hagman, "Hardware companions?-What online AIBO discussion forums reveal about the human-robotic relationship," Proceedings of the CHI 2003 Conference on Human Factors in Computing Systems. New York: ACM, 2003.

[18] S. Turkle, W. Taggart, C. D. Kidd, and O. Dasté, "Relational artifacts with children and elders: the complexities of cybercompanionship," Connection Science, vol. 18, pp. 347-361, 2006.

[19] LIREC, http://www.lirec.org, 2008.

[20] M. D. Klinnert, R. N. Emde, P. Butterfield, and J. J. Campos, "Social referencing: The infant's use of emotional signals from a friendly adult with mother present.," Developmental Psychology, vol. 22, pp. 427-432, 1986.

[22] K.L. Koay, D. S. Syrdal , M.L. Walters , and K. Dautenhahn . Living with Robots: Investigating the Habituation Effect in Participants? Preferences during a Longitudinal Human-Robot Interaction. Proceedings of the IEEE International Symposium on Robot and Human Interactive Communication (Ro-Man 2078, Jeju Island, Korea, 26-29 August 200, 564-569.

[22] D. S. Syrdal, N. R. Otero, and K. Dautenhahn, "Video Prototyping in Human-Robot Interaction: Results from a Qualitative Study," Proceedings of the 2008 European Conference on Cognitive Ergonomics, 2008.

[23] A. Light, "Adding method to meaning: A Technique for exploring peoples' experiences with technology," Behaviour \& Information Technology, vol. 25, pp. 175-187, 2006.

[24] N. Pidgeon and K. Henwood, "Using grounded theory in psychological research," in Doing Qualitative Analysis in Psychology, N. Hayes, Ed. London: Psychology Press, 1997, pp. 245-274.

[25] J.-Y. Sung, R. E. Grinter, H. I. Christensen, and L. Guo, "Housewives or technophiles? understanding domestic robot owners," in Proceedings of the 3rd ACM/IEEE international conference on Human robot interaction. Amsterdam, The Netherlands: ACM, 2008.

[26] Grudin, J. \& Pruitt, J . Personas, participatory design, and product development: An infrastructure for engagement. Proc. PDC 2002, $144-161,2002$

[27] A.R. Chatley, K.Dautenhahn, M.L. Walters, D.S. Syrdal and B. Christianson. Theatre as a discussion tool in human-hobot interaction experiments. Proceedings The Third International Conference on Advances in Computer-Human Interactions ACHI 2010, February 1016, 2010 - St. Maarten, Netherlands Antilles, IEEE Press, pages 73 78,2010

[28] M. Jacobsson, "Play, Belief and Stories about Robots: A Case Study of a Pleo Blogging Community," Proceedings of the IEEE International Symposium on Robot and Human Interactive Communication (Ro-Man 2009), 2009. 